\title{
Metabolic mapping of $A_{3}$ adenosine receptor agonist MRS5980
}

\author{
Zhong-Ze Fang ${ }^{\mathrm{a}, \mathrm{c}}$, Dilip K. Tosh ${ }^{\mathrm{b}}$, Naoki Tanaka ${ }^{\mathrm{a}}$, Haina Wang ${ }^{\mathrm{a}, \mathrm{d}}$, Kristopher W. Krausz ${ }^{\mathrm{a}}$, \\ Robert O'Connor ${ }^{b}$, Kenneth A. Jacobson ${ }^{b,}{ }^{,}$, and Frank J. Gonzalez ${ }^{a,{ }^{*}}$ \\ aLaboratory of Metabolism, Center for Cancer Research, National Cancer Institute, National \\ Institutes of Health, Bethesda, MD 20892, USA \\ bMolecular Recognition Section, Laboratory of Bioorganic Chemistry, National Institute of \\ Diabetes and Digestive and Kidney Diseases, National Institutes of Health, Bethesda, MD \\ 20892-0810, USA \\ 'Department of Toxicology, School of Public Health, Tianjin Medical University, Tianjin, 300070, \\ China
}

\section{Abstract}

(1S,2R,3S,4R,5S)-4-(2-((5-Chlorothiophen-2-yl)ethynyl)-6-(methylamino)-9H-purin-9-yl)-2,3dihydroxy- $N$-methylbicyclo[3.1.0]hexane-1-carboxamide (MRS5980) is an $\mathrm{A}_{3} \mathrm{AR}$ selective agonist containing multiple receptor affinity- and selectivity-enhancing modifications and a therapeutic candidate drug for many inflammatory diseases. Metabolism-related poor pharmacokinetic behavior and toxicities are a major reason of drug R\&D failure. Metabolomics with UPLC-MS was employed to profile the metabolism of MRS5980 and MRS5980-induced disruption of endogenous compounds. Recombinant drug-metabolizing enzymes screening experiment were used to determine the enzymes involved in MRS5980 metabolism. Analysis of lipid metabolism-related genes was performed to investigate the reason for MRS5980-induced lipid metabolic disorders. Unsupervised principal components analysis separated the control and MRS5980 treatment group in feces, urine, and liver samples, but not in bile and serum. The major ions mainly contributing to the separation for feces and urine were oxidized MRS5980, glutathione (GSH) conjugates and cysteine conjugate (degradation product of the GSH conjugates) of MRS5980. The major ions contributing to the group separation of liver samples were phosphatidylcholines. In vitro incubation experiments showed the major involvement of CYP3A enzymes in the oxidative metabolism of MRS5980 and direct GSH reactivity of MRS5980. The electrophilic attack by MRS5980 is a minor pathway and did not alter GSH levels in liver or liver histology, and thus may be of minor clinical consequence. Gene expression analysis further showed decreased expression of PC biosynthetic genes choline kinase a and $b$, which further

\footnotetext{
"Corresponding authors: Frank J. Gonzalez, Laboratory of Metabolism, Center for Cancer Research, National Cancer Institute, National Institutes of Health, Bethesda, MD, 20892, gonzalef@mail.nih.gov; Kenneth A. Jacobson, Molecular Recognition Section, Laboratory of Bioorganic Chemistry, National Institute of Diabetes and Digestive and Kidney Diseases, National Institutes of Health, Bldg. 8A, Rm. 81A-19, Bethesda, MD 20892-0810, kajacobs@helix.nih.gov.

dPresent address: College of Pharmaceutical Sciences, Shandong University, Shandong, 250012, China

Conflicts of Interest: The authors report no conflict of interest.

Publisher's Disclaimer: This is a PDF file of an unedited manuscript that has been accepted for publication. As a service to our customers we are providing this early version of the manuscript. The manuscript will undergo copyediting, typesetting, and review of the resulting proof before it is published in its final citable form. Please note that during the production process errors may be discovered which could affect the content, and all legal disclaimers that apply to the journal pertain.
} 
accelerated conversion of lysophosphatidylcholine to phosphatidylcholines through increasing the expression of lysophosphatidylcholine acyltransferase 3. These data will be useful to guide rational design of drugs targeting $\mathrm{A}_{3} \mathrm{AR}$, considering efficacy, metabolic elimination, and electrophilic reactivity.

\section{Keywords}

$\mathrm{A}_{3}$ adenosine receptor (AR); MRS5980; metabolomics; electrophilies

\section{Introduction}

Drug efficiency, toxicity, and metabolic elimination are the major properties determining whether a drug candidate can successfully enter clinical trials [1]. Through combining in vitro incubation mixtures and in vivo animal models, the metabolic pathways of drugs and drug candidates have been elucidated, such as ifosfamide [2], noscapine [3] and aryl hydrocarbon receptor antagonist GNF-351 [4]. Endogenous metabolomics can be used to phenotype the alteration of endogenous substances, which can directly reflect the disruption of metabolic pathways by the drug and serve as biomarkers for efficacy and toxicity. For example, serum metabolomics was employed to explain the adverse effects of gemfibrozil through analyzing the alteration of lysophosphatidylcholine and bile acid components in gemfibrozil-treated mice[5].

The $\mathrm{A}_{3}$ adenosine receptor (AR), a $\mathrm{G}$ protein-coupled receptor, is a therapeutic target for many diseases (e.g., hepatocellular carcinoma, autoimmune inflammatory diseases, chronic neuropathic pain, etc.), and development of $\mathrm{A}_{3} \mathrm{AR}$ selective agonists as drug candidates has been a major goal to treat these diseases [6,7]. $(1 S, 2 R, 3 S, 4 R, 5 S)-4-(2-((5-C h l o r o t h i o p h e n-2-$ yl)ethynyl)-6-(methylamino)-9H-purin-9-yl)-2,3-dihydroxy- $N$ methylbicyclo[3.1.0]hexane-1-carboxamide (MRS5980) is a recently developed $\mathrm{A}_{3} \mathrm{AR}$ selective agonist that contains multiple receptor affinity- and selectivity-enhancing modifications [8]. This nucleoside derivative contains a rigid ribose carbocyclic substitution ([3.1.0]bicyclohexane) that maintains a receptor-preferred conformation. The 2-arylethynyl substituent of sterically constrained nucleoside analogues is predicted to induce an adjustment of the position of the second transmembrane helical domain of the $\mathrm{A}_{3} \mathrm{AR}$, which contributes to its lack of affinity at the $\mathrm{A}_{2 \mathrm{~A}} \mathrm{AR}$ [8]. The present study used metabolomics to evaluate the MRS5980-body metabolism and interactions, including the metabolic elimination by the body of MRS5980, electrophilic reactivity and influence on lipid profiles of MRS5980 in the body.

\section{Materials and methods}

\subsection{Chemicals and reagents}

MRS5980 was synthesized as described [8]. $\beta$-Nicotinamide adenine dinucleotide $2^{\prime}$ phosphate reduced tetrasodium salt (NADPH) and reduced glutathione were purchased from Sigma-Aldrich (St. Louis, MO). All other reagents were of the highest grade commercially available. Livers from untreated 6- to 8-week-old male C57BL/6J mice were used to prepare mouse liver microsomes (MLMs) as previously described [4]. Human liver microsomes 
(HLMs), recombinant cytochrome P450 (CYP) 1A1, 1A2, 1B1, 2A6, 2B6, 2C9, 2C9*2, $2 \mathrm{C} 9 * 3,2 \mathrm{C} 19,2 \mathrm{D} 6,2 \mathrm{E} 1,3 \mathrm{~A} 4$, and 3A5 were purchased from BD Gentest Corp. (Woburn, MA, USA).

\subsection{Animal treatment and samples preparation}

Ten male C57BL/6J (6- to 8-week-old) mice were divided into control group (n=5) and MRS5980-treated group ( $\mathrm{n}=5)$. MRS5980 dissolved in corn oil was given by oral gavage at a dose of $50 \mathrm{mg} / \mathrm{kg}$ body weight, and control mice were treated with corn oil only. All studies involving animals were under protocols following Animal Research Reporting of In Vivo Experiments (ARRIVE) guidelines. Mouse handling was performed in accordance with an animal study protocol approved by the National Cancer Institute Animal Care and Use Committee. The mice were maintained under a standard $12 \mathrm{~h}$ light/12 $\mathrm{h}$ dark cycle with water and chow provided ad libitum. Urine and feces samples ( $24 \mathrm{~h})$ were collected using metabolic cages (Metabowls, Jencons Scientific USA, Bridgeville, PA), and blood samples were collected into BD microtainer serum separator tubes (Franklin Lakes, NJ) by retroorbital bleeding. Serum was made through 15 min centrifugation at $8000 \times \mathrm{g}$. After killing by $\mathrm{CO}_{2}$ asphyxiation, gallbladder and liver samples were harvested, and a small section of liver excised for histological analysis. All the samples were stored at $-80^{\circ} \mathrm{C}$ until analysis. For sample preparation, $5 \mu \mathrm{l}$ of serum was diluted with $195 \mu \mathrm{l}$ of $66 \%$ aqueous acetonitrile containing $5 \mu \mathrm{M}$ chlorpropamide. Urine samples were prepared by mixing $5 \mu \mathrm{l}$ of urine with $195 \mu \mathrm{l}$ of $50 \%$ aqueous acetonitrile containing $5 \mu \mathrm{M}$ chlorpropamide. The feces were pulverized and 1:20 50\% aqueous acetonitrile ( $5 \mu \mathrm{M}$ chlorpropamide) was added for extraction followed by centrifugation. The homogenates of liver were prepared and diluted with 1:20 50\% aqueous acetonitrile ( $5 \mu \mathrm{M}$ chlorpropamide). All samples were centrifuged at $14,000 \times \mathrm{g}$ for $15 \mathrm{~min}$, and $5 \mu \mathrm{l}$ aliquot of the supernatants was injected into a Waters UPLCESI-QTOFMS system (Waters Corporation, Milford, MA).

\subsection{Analysis of liver histology}

The analysis of liver histology was performed as previously described [9]. In brief, small blocks of liver tissues from all mice were immediately fixed in 10\% neutral formalin and embedded in paraffin. Sections ( $4 \mu \mathrm{m}$ thick) were stained by the hematoxylin and eosin or Sirius red method.

\subsection{In vitro incubation reaction to analyze the metabolic pathway of MRS5980}

For MLM and HLM, the incubation system contained $50 \mathrm{mM}$ tris- $\mathrm{HCl}$ buffer solution $(\mathrm{pH}=$ 7.4), $0.5 \mathrm{mg} \mathrm{mL}^{-1} \mathrm{HLM}$ or MLM, $5 \mathrm{mM} \mathrm{MgCl}_{2}, 100 \mu \mathrm{M}$ MRS5980 dissolved in DMSO, $2.5 \mathrm{mM}$ glutathione (GSH) and $1 \mathrm{mM}$ freshly prepared NADPH. The incubation mixtures without GSH, MLM (or HLM), NADPH and MRS5980 were used as the control. After $0.5 \mathrm{~h}$ incubation at $37^{\circ} \mathrm{C}$, the reactions were stopped using $200 \mu \mathrm{L}$ cold $50 \%$ aqueous methanol containing $5 \mu \mathrm{M}$ chlorpropamide. After centrifuging at $14000 \times \mathrm{g}$ for $20 \mathrm{~min}$, a $5 \mu \mathrm{L}$ aliquot of the supernatant was injected into a UPLC-ESI-QTOFMS. The in vitro incubation system for recombinant phase I enzymes was similar to the microsomal incubation system just without GSH. Recombinant CYPs ( 2 pmol) were incubated with $100 \mu$ M of MRS5980. The reaction time was $30 \mathrm{~min}$ and metabolites were analyzed using UPLC-ESIQTOFMS. 


\subsection{UPLC-ESI-QTOFMS analysis}

An Acquity C18 BEH UPLC column (Waters Corporation) was employed to separate components in serum, urine, feces and microsomal incubation samples. The mobile phase consisted of water containing $0.1 \%$ formic acid (A) and acetonitrile containing $0.1 \%$ formic acid (B). The following gradient condition was used: $100 \%$ A for $0.5 \mathrm{~min}$, increased to $100 \% \mathrm{~B}$ over the next $7.5 \mathrm{~min}$ and returned to $100 \% \mathrm{~A}$ in last $2 \mathrm{~min}$. The flow rate of the mobile phase was set at $0.5 \mathrm{ml} / \mathrm{min}$. Data were collected in the positive ion mode on a Waters Q-Tof Premier mass spectrometer, which was operated in full-scan mode at 50-850 $\mathrm{m} / \mathrm{z}$. Nitrogen was used as both cone gas $(50 \mathrm{l} / \mathrm{h})$ and desolvation gas $(600 \mathrm{l} / \mathrm{h})$. Source desolvation temperatures were set at $120^{\circ} \mathrm{C}$ and $350{ }^{\circ} \mathrm{C}$, respectively. The capillary voltage and cone voltage were 3000 and $20 \mathrm{~V}$, respectively. The structures of metabolites were elucidated by tandem MS fragmentography with collision energies ranging from 15 to 40 $\mathrm{eV}$. For the determination of GSH, a GSH standard was used to identify the retention time of GSH, and the relevant abundance of GSH was compared.

\subsection{Data processing and multivariate data analysis (MDA)}

MarkerLynx software (Waters Corp.) was used to deconvolute the chromatographic and mass spectrometric data. A multivariate data matrix containing information on sample identity, ion identity $\left(\mathrm{R}_{\mathrm{t}}\right.$ and $\left.\mathrm{m} / \mathrm{z}\right)$, and ion abundance was generated through centroiding, deisotoping, filtering, peak recognition, and integration. The data matrix was further analyzed using SIMCA-13.0 software (Umetrics, Kinnelon, NJ). Principle components analysis (PCA) was used to examine the separation of control group and MRS5980-treated group. Potential metabolites were identified by analyzing the ions contributing to the separation of sample groups in the loading scatter plots.

\subsection{Quantitative polymerase chain reaction (qPCR) analysis}

Quantitative polymerase chain reaction (qPCR) analysis was carried out as previously described [10]. Total liver RNA was extracted using TRIzol reagent (Invitrogen, Carlsbad, CA), and cDNA was generated from $1 \mu \mathrm{g}$ RNA with a SuperScript II ${ }^{\mathrm{TM}}$ Reverse Transcriptase kit and random oligonucleotides (Invitrogen, Grand Island, NY). Quantitative PCR (qPCR) was performed using SYBR green PCR master mix and ABI Prism 7900HT Sequence Detection System (Applied Biosystems, Foster City, CA). The primer pairs were designed using qPrimerDepot, and messenger RNA quantitation was performed using the comparative cycle threshold (CT) method. The expression of mRNA was normalized to mouse $\beta$-actin.

\subsection{Statistical analysis}

The experimental data were presented as mean \pm S.E.M. Comparisons between two groups were performed using a two-tailed unpaired student's $t$ test. 


\section{Results}

\subsection{Metabolomics analysis to find biomarkers for mice exposure to MRS5980}

The chromatographic behavior of MRS5980 showed elution at $5.6 \mathrm{~min}$ (Fig. 1A), and MRS5980 showed doublet m/z 459.104 and 461.102 ions (Fig. 1B). MS was performed in the positive-ion mode, because compounds containing halogens (especially chlorine and bromine) can produce very distinct isotope peaks [11]. The MS/MS spectrum of MRS5980 $(\mathrm{m} / \mathrm{z}=459.104)$ showed the major fragment ions 428 and 290, and the corresponding fragmentation patterns were also given (Fig. 1C). UPLC-ESI-QTOFMS analysis coupled with MDA was employed to profile the metabolome for urine, feces, liver, bile, and serum. Unsupervised principal components analysis (PCA) separates the control and MRS5980 treatment groups from feces, urine, and liver samples, but not from bile and serum samples (Fig. 2). The major ions contributing to the separation of urine and feces samples contained (P1, P2, P3, P4) and (P1,P2,P3,P5,P6), respectively (Table 1), and the major ions contributing to the separation of liver samples were P7-P10. The relative abundance of these ions in the control and MRS5980 treatment groups was given in Fig. 3.

\subsection{MS/MS identification of detected biomarkers}

$\mathrm{P} 1$ ion has the molecular ion 459.105 in the positive mode and was identified as the parent compound MRS5980 with a mass error of $9.6 \mathrm{ppm}$. The molecular ions of P2 and P3 are 16 Da higher than P1 (MRS5980), indicating that they are oxidized metabolites of MRS5980. Compared with the proposed formula $\left(\mathrm{C}_{20} \mathrm{H}_{19} \mathrm{ClN}_{6} \mathrm{O}_{4} \mathrm{~S}\right)$, the mass errors of $\mathrm{P} 2$ and $\mathrm{P} 3$ were 1.1 and $3.0 \mathrm{ppm}$. The MS/MS fragment of $\mathrm{P} 2$ at 276 indicated that an oxidation reaction occurred in the 290 fragment of the parent compound, and the possible oxidation site might be the $N^{6}$-methyl group, and the possible fragmentation mechanism (Fig. 4). P3 has the major fragment 290, indicating the occurrence of oxidation in another part of MRS5980. P4 has a molecular ion of 766.186, which closely corresponds to the molecular formula $\mathrm{C}_{30} \mathrm{H}_{36} \mathrm{ClN}_{9} \mathrm{O}_{9} \mathrm{~S}_{2}$, indicating a direct conjugation of MRS5980 with reduced glutathione. The MS/MS fragments gave typical GSH fragments at 637 (neutral loss of 129) and 493 (neutral loss of 273), further demonstrating the formation of GSH conjugate(s). According to alkyne reactivity in published reports [12], this is a GSH conjugate of MRS5980 at the triple bond. P5 (6) appeared to be a cysteine conjugate of MRS5980 according the MS/MS fragments and the proposed fragmentation mechanism (Fig. 4). P7-P10 exhibited the molecular ions 816.541, 790.531, 706.507, and 680.487 (Fig. 5), respectively. Based on the metabolomics database (http://www.hmdb.ca/), these ions were assumed to be lipid components. The MS/MS spectra gave the same fragment at 184, which was a typical fragment of phosphatidylcholine (PC) and lysophosphatidylcholine (LPC). Given the relatively high molecular weight, these components seemed to be PC components.

\subsection{CYP3A4/5 was the major enzyme responsible for the elimination of MRS5980}

An in vitro incubation system with human liver microsomes (HLMs) and mouse liver microsomes (MLMs) was initially used to model the in vivo metabolic behavior of MRS5980. Unsupervised principal components analysis (PCA) separated the MLM complete incubation system and MLM incubation system without NADPH, MLM, GSH and substrate (Fig. 6). The oxidized metabolites of MRS5980 (P2 and P3) appeared in the 
complete MLM incubation system and also upon incubation without GSH, but not in the incubation system without NADPH, liver microsomes, and substrate (Fig. 7), indicating that the formation of metabolites was NADPH-, microsome-, and substrate-dependent. The trend plots (Fig. 7) also showed that the GSH conjugate P4 appeared in the complete MLM incubation system and the mixture without NADPH and microsomes, but not in the incubation system without GSH and substrate. Therefore, formation of the GSH conjugates did not require involvement of drug-metabolizing enzymes, thus indicating a direct attack of GSH towards MRS5980. Another GSH conjugate (P11) with the same molecular ion $\left(\mathrm{R}_{\mathrm{t}}=4.37 \mathrm{~min}\right.$ ) appeared (Fig. 7). It has the same MS/MS fragment with P4. The formation of metabolites was also observed in the HLM incubation system (Fig. 8), and GSH conjugates P4 (5) can also form without the involvement of enzymes and NADPH. Additionally, a new GSH conjugate $\mathrm{P} 12\left(\mathrm{R}_{\mathrm{t}}=4.97 \mathrm{~min}, \mathrm{~m} / \mathrm{z}=764.175\right)$ was detected in HLM incubation system, and the formation of this conjugate is dependent on the presence of NADPH, indicating the involvement of bioactivation in the formation of this conjugate. Recombinant phase I drugmetabolizing enzymes screening showed that CYP3A4/5 exhibited stronger catalytic activity than other CYP isoforms towards the formation of MRS5980's oxidized products P2 and P3 (Fig. 6C).

\subsection{In vivo effects of MRS5980 on liver histology, GSH and lipid genes involved in lipid metabolism}

Treatment with MRS5980 did not affect the GSH levels in liver (Fig. 9A) or liver histology (Fig. 9B). The expression of genes related to lipid metabolism was investigated and the results showed that the treatment with MRS5980 can inhibit the expression of choline kinase a (Chka) and b (Chkb) by $41 \%$ and $28 \%$ (not statistically significant), respectively, and significantly elevate the expression of Lpcat3 mRNA (Fig. 9C). Treatment with MRS5980 did not show any influence on the expression of Chpt1, Pcyt1b, Lcat, Lpcat1, Lpcat2, and Lpcat4 (Fig. 9C).

\section{Discussion}

Developing safer drugs and drug candidates has been a major goal of the pharmaceutical industry, and its importance has been intensified by the withdrawal of many drugs (e.g., Ximelagatran, Rofecoxib, Lumiracoxib, etc.) from the market and a decrease of new chemical entities (NCEs) introduced into the market [13]. In preclinical efficacy testing, in the chronic constriction injury (CCI, sciatic nerve) model in mice, the $\mathrm{ED}_{50}$ value of MRS5980 at its peak effect following oral administration was $0.34 \mathrm{mg} / \mathrm{kg}(0.73 \mathrm{~mol} / \mathrm{kg}$; $\mathrm{n}=5$ ) [6]. On a molar dose basis, this is more potent than morphine, amitriptyline or gabapentin in the same model [14]. Another potential advantage of selective $\mathrm{A}_{3} \mathrm{AR}$ agonists over opiates for control of chronic pain is that tolerance was not observed in initial testing. An infusion of a closely related 2-arylalkynyl adenosine derivative MRS5698 ( $(1 S, 2 R, 3 S$, $4 R, 5 S)$-4-(6-((3-chlorobenzyl)amino)-2-((3,4-difluorophenyl)ethynyl)-9H-purin-9-yl)-2,3dihydroxy- $N$-methylbicyclo[3.1.0]hexane-1-carboxamide) of selectivity comparable to MRS5980, when infused over 6 days via subcutaneous mini-pump, did not lose its antinociceptive effects [15]. Thus, $\mathrm{A}_{3} \mathrm{AR}$ agonists such as MRS5980 show promise as new agents for control of chronic, but not acute pain. 
Electrophilic groups either located in the parent compounds or formed via bioactivation can attack macromolecules in vivo (e.g., protein, nucleic acids, etc.) through covalent binding, or consume the reducing substances (e.g. glutathione, etc.). Many structural alerts were reported previously, such as those associated with anilines, thiophenes, furans, formamides, thioamides, hydrazines and sulfonylureas [16]. Depending on its substitution, a triple bond has been widely reported to be an important structural alert, and many compounds with this structure have been demonstrated to exhibit potential toxic risks. For example, the triple bond in the HIV therapy drug (S)-5,6-difluoro-4-cyclopropylethynyl-4-trifluoromethyl-3,4dihydro-2-(1H)-quinazolinone (DPC963, Efavirenz) can form through multiple bioactivation pathways reactive intermediates, which can be trapped by reduced glutathione (GSH) [17]. The triple bond in the epidermal growth factor receptor inhibitor erlotinib, which has a highly reactive monosubstituted alkyne, can directly attack GSH without catalytic transformation by drug-metabolizing enzymes (DMEs) [12]. In the present study, a GSH conjugation was observed, and GSH conjugates of MRS5980 detected in the urine and feces from MRS5980-treated mice, indicating an electrophilic attack of MRS5980 towards the GSH system. Furthermore, in vitro incubation experiments demonstrated that the formation of GSH conjugates was independent of catalysis mediated by DMEs, i.e. bioactivation. Following previous studies, the GSH conjugates were proposed to be cis- and trans- GSH conjugates of MRS5980 at the triple bond [12]. Additionally, degradation products of MRS5980-GSH conjugates, Cys-MRS5980, were also found in urine and feces. It should be noted that a new bioactivation-catalyzed GSH conjugate appeared in HLM incubation system, indicating a species difference in bioactivation of MRS5980. During $24 \mathrm{~h}$ drug exposure to MRS5980 (50 mg/kg, 50-fold higher than a fully efficacious dose in reducing chronic neuropathic pain [7]), the liver histology remains normal, and no alteration of GSH and GSSG levels in liver was observed. This indicates that there are no significant toxic effects due to potential GSH fluctuation resulting from the reaction of GSH and MRS5980. However, future study will be needed to fully evaluate the influence of MRS5980 treatment towards the GSH system. Although liver histology was not strikingly affected by MRS5980, the data suggest that liver function should be examined after prolonged exposure to this compound.

Besides the GSH attacking effect, MRS5980 also influenced lipid homeostasis, as shown by MRS5980-induced reduction of PC components. Gene expression analysis showed that treatment with MRS5980 inhibited the expression of choline kinase a (Chka) and b (Chkb), two key genes catalyzing the biosynthesis of PC [18]. The reduction of PC levels accelerated the conversion from LPC to PC, as demonstrated by increased expression of lysophosphatidylcholine acyltransferase 3 (Lpcat3), which is a key gene responsible for the conversion of LPC to PC. PCs are considered to be biologically important molecules and to exhibit important in vivo functions, including anti-inflammatory effects [19] and the attenuation of oxidative stress [20]. Altered PC levels have been detected in various diseases or toxicity models. For example, the PC content was reduced by $70 \%$ in ulcerative colitis (UC) [21]. In the toxicity model induced by treatment with trichloroethylene, trichloroacetate and dichloroacetate, the PC components significantly increased [10]. Therefore, the final toxicity results of the influence of MRS5980 towards PC components levels should be investigated in detail. 
CYP3A was demonstrated to the most active enzyme involved in the metabolism of MRS5980. Therefore, CYP3A-mediated drug metabolic elimination of MRS5980 could decrease the concentration of MRS5980 exhibiting electrophilic reactivity and influence on the lipid profile. The CYP3A-related drug-drug interaction (DDI) should be followed in case of co-administration of MRS5980 with CYP3A substrates (e.g., midazolam, nifedipine, etc.), inhibitors (e.g., ketoconazole, noscapine, etc.) or inducers (e.g., rifaximin, rifampicin, etc.) [22-27]. Additionally, individual differences in CYP3A activity could also affect the exposure of MRS5980, and the final in vivo results (GSH reactivity and influence on lipid homeostasis).

In conclusion, metabolomics was applied to study metabolism of the candidate drug, $\mathrm{A}_{3} \mathrm{AR}$ agonist MRS5980. The balance between efficacy, metabolic elimination, and electrophilic reactivity and the role of altered lipids and how they influence properties of $\mathrm{A}_{3} \mathrm{AR}$-targeting drugs should be considered for future studies.

\section{Acknowledgments}

This study was funded by the Intramural Research Programs of the Center for Cancer Research, National Cancer Institute and the National Institute of Diabetes and Digestive and Kidney Diseases, National Institutes of Health, the National Natural Science Foundation of China (No. 81202586), and Tianjin Project of Thousand Youth Talents.

\section{References}

1. Fang ZZ, Gonzalez FJ. LC-MS-based metabolomics: an update. Arch Toxicol. 2014; 88:1491-1502. [PubMed: 24710571]

2. Li F, Patterson AD, Hofer CC, Krausz KW, Gonzalez FJ, Idle JR. Comparative metabolism of cyclophosphamide and ifosfamide in the mouse using UPLC-ESI-QTOFMS-based metabolomics. Biochem Pharmacol. 2010; 80:1063-1074. [PubMed: 20541539]

3. Fang ZZ, Krausz KW, Li F, Cheng J, Tanaka N, Gonzalez FJ. Metabolic map and bioactivation of the anti-tumour drug noscapine. Br J Pharmacol. 2012; 167:1271-86. [PubMed: 22671862]

4. Fang ZZ, Krausz KW, Nagaoka K, Tanaka N, Gowda K, Amin SG, Perdew GH, Gonzalez FJ. In vivo effects of the pure aryl hydrocarbon receptor antagonist GNF-351 after oral administration are limited to the gastrointestinal tract. Br J Pharmacol. 2014; 171:1735-1746. [PubMed: 24417285]

5. Liu A, Krausz KW, Fang ZZ, Brocker C, Qu A, Gonzalez FJ. Gemfibrozil disrupts lysophosphatidylcholine and bile acid homeostasis via PPARa and its relevance to hepatotoxicity. Arch Toxicol. 2014; 88:983-996. [PubMed: 24385052]

6. Tosh DK, Crane S, Chen Z, Paoletta S, Gao ZG, Gizewski E, Auchampach JA, Salvemini D, Jacobson KA. Rigidified $\mathrm{A}_{3}$ adenosine receptor agonists: 1-Deaza modification maintains high in vivo efficacy. ACS Med Chem Lett. in press. 10.1021/acsmedchemlett.5b00150

7. Borea PA, Varani K, Vincenzi F, Baraldi PG, Tabrizi MA, Merighi S, Gessi S. The A3 adenosine receptor: history and perspectives. Pharmacol Rev. 2015; 67(1):74-102. [PubMed: 25387804]

8. Tosh DK, Finley A, Paoletta S, Moss SM, Gao ZG, Gizewski ET, Auchampach JA, Salvemini D, Jacobson KA. In vivo phenotypic screening for treating chronic neuropathic pain: modification of $\mathrm{C} 2$-arylethynyl group of conformationally constrained $\mathrm{A}_{3}$ adenosine receptor agonists. $\mathrm{J}$ Med Chem. 2014; 57:9901-9914. [PubMed: 25422861]

9. Cheng J, Fang ZZ, Kim JH, Krausz KW, Tanaka N, Chiang JY, Gonzalez FJ. Intestinal CYP3A4 protects against lithocholic acid-induced hepatotoxicity in intestine-specific VDR-deficient mice. $\mathrm{J}$ Lipid Res. 2014; 55:455-465. [PubMed: 24343899]

10. Fang ZZ, Krausz KW, Tanaka N, Li F, Qu A, Idle JR, Gonzalez FJ. Metabolomics reveals trichloroacetate as a major contributor to trichloroethylene-induced metabolic alterations in mouse urine and serum. Arch Toxicol. 2013; 87:1975-1987. [PubMed: 23575800] 
11. Song JH, Fang ZZ, Zhu LL, Cao YF, Hu CM, Ge GB, Zhao DW. Glucuronidation of the broadspectrum antiviral drug arbidol by UGT isoforms. J Pharm Pharmacol. 2013; 65:521-527. [PubMed: 23488780]

12. Li X, Kamenecka TM, Cameron MD. Cytochrome P450-mediated bioactivation of the epidermal growth factor receptor inhibitor erlotinib to a reactive electrophile. Drug Metab Dispos. 2010; 38:1238-1245. [PubMed: 20382753]

13. Edwards PJ, Sturino C. Managing the liabilities arising from structural alerts: a safe philosophy for medicinal chemists. Cur Med Chem. 2011; 18:3116-35.

14. Chen Z, Janes K, Chen C, Doyle T, Tosh DK, Jacobson KA, Salvemini D. Controlling murine and rat chronic pain through $\mathrm{A}_{3}$ adenosine receptor activation. FASEB J. 2012; 26:1855-1865. [PubMed: 22345405]

15. Little JW, Ford A, Symons-Liguori AM, Chen Z, Janes K, Doyle T, Xie J, Luongo L, Tosh DK, Maione S, Bannister K, Dickenson A, Vanderah TW, Porreca F, Jacobson KA, Salvemini D. Endogenous adenosine $\mathrm{A}_{3}$ receptor activation selectively alleviates persistent pain states. Brain. 2015; 138:28-35. [PubMed: 25414036]

16. Walsh JS, Miwa GT. Bioactivation of drugs: risk and drug design. Annu Rev Pharmacol Toxicol. 2011; 51:145-167. [PubMed: 21210745]

17. Chen H, Chen W, Gan LS, Mutlib AE. Metabolism of (S)-5,6-difluoro-4-cyclopropylethynyl-4trifluoromethyl-3, 4-dihydro-2 $(1 \mathrm{H})$-quinazolinone, a non-nucleoside reverse transcriptase inhibitor, in human liver microsomes. Metabolic activation and enzyme kinetics. Drug Metab Dispos. 2003; 31:122-132. [PubMed: 12485961]

18. Matsubara T, Tanaka N, Patterson AD, Cho JY, Krausz KW, Gonzalez FJ. Lithocholic acid disrupts phospholipid and sphingolipid homeostasis leading to cholestasis in mice. Hepatology. 2011; 53:1282-1293. [PubMed: 21480330]

19. Jung YY, Nam Y, Park YS, Lee HS, Hong SA, Kim BK, Park ES, Chung YH, Jeong JH. Protective effect of phosphatidylcholine on lipopolysaccharide-induced acute inflammation in multiple organ injury. Korean J Physiol Pharmacol. 2013; 17:209-216. [PubMed: 23776397]

20. Okiyama W, Tanaka N, Nakajima T, Tanaka E, Kiyosawa K, Gonzalez FJ, Aoyama T. Polyenephosphatidylcholine prevents alcoholic liver disease in PPARalpha-null mice through attenuation of increases in oxidative stress. J Hepatol. 2009; 50:1236-1246. [PubMed: 19398233]

21. Stremmel W, Ehehalt R, Staffer S, Stoffels S, Mohr A, Karner M, Braun A. Mucosal protection by phosphatidylcholine. Dig Dis. 2012; 30(Suppl 3):85-91. [PubMed: 23295697]

22. Chien JY, Lucksiri A, Ernest CS, Gorski JC, Wrighton SA, Hall SD. Stochastic prediction of CYP3A-mediated inhibition of midazolam clearance by ketoconazole. Drug Metab Dispos. 2006; 34:1208-1219. [PubMed: 16611859]

23. Quinney SK, Mohamed AN, Hebert MF, Haas DM, Clark S, Umans JG, Caritis SN, Li L. A semimechanistic metabolism model of CYP3A substrates in pregnancy: predicting changes in midazolam and nifedipine pharmacokinetics. CPT Pharmacometrics Syst Pharmacol. 2012; 1:e2. [PubMed: 23835882]

24. Salem AH, Yang J, Graham A, Patnaik A, Holen K, Pradhan R, Xiong H. Effect of coadministration of ketoconazole, a strong CYP3A inhibitor, on the pharmacokinetics, safety and tolerability of navitoclax, a first-in-class oral Bcl-2 family inhibitor in cancer patients. Anticancer Res. 2014; 34:2001-2006. [PubMed: 24692738]

25. Fang ZZ, Zhang YY, Ge GB, Huo H, Liang SC, Yang L. Time-dependent inhibition (TDI) of CYP3A4 and CYP2C9 by noscapine potentially explains clinical noscapine-warfarin interaction. Br J Clin Pharmacol. 2010; 69:193-199. [PubMed: 20233183]

26. Ma X, Shah YM, Guo GL, Wang T, Krausz KW, Idle JR, Gonzalez FJ. Rifaximin is a gut-specific human pregnane X receptor activator. J Pharmacol Exp Ther. 2007; 322:391-398. [PubMed: 17442842]

27. Song X, Xie M, Zhang H, Li Y, Sachdeva K, Yan B. The pregnane X receptor binds to response elements in a genomic context-dependent manner, and PXR activator rifampicin selectively alters the binding among target genes. Drug Metab Dispos. 2004; 32:35-42. [PubMed: 14709618] 


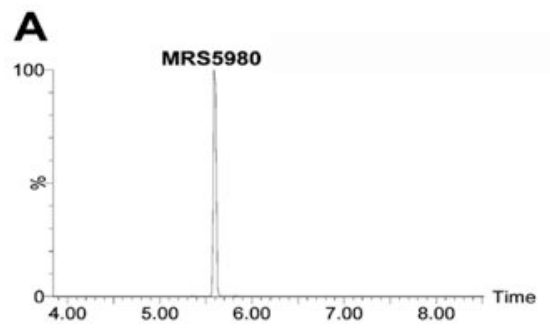

B

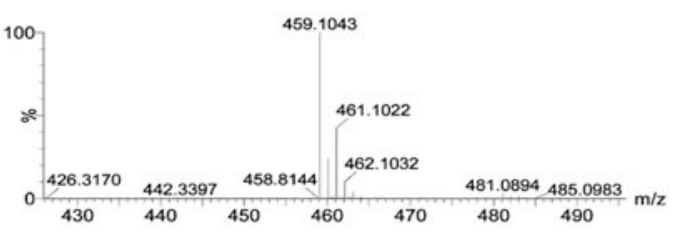

C

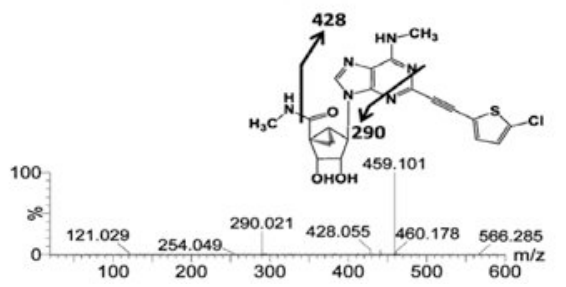

Fig. 1.

Determination of chromatography behavior of MRS5980, including retention time (A), MS spectrum (B), and MS/MS fragmentation (C). 

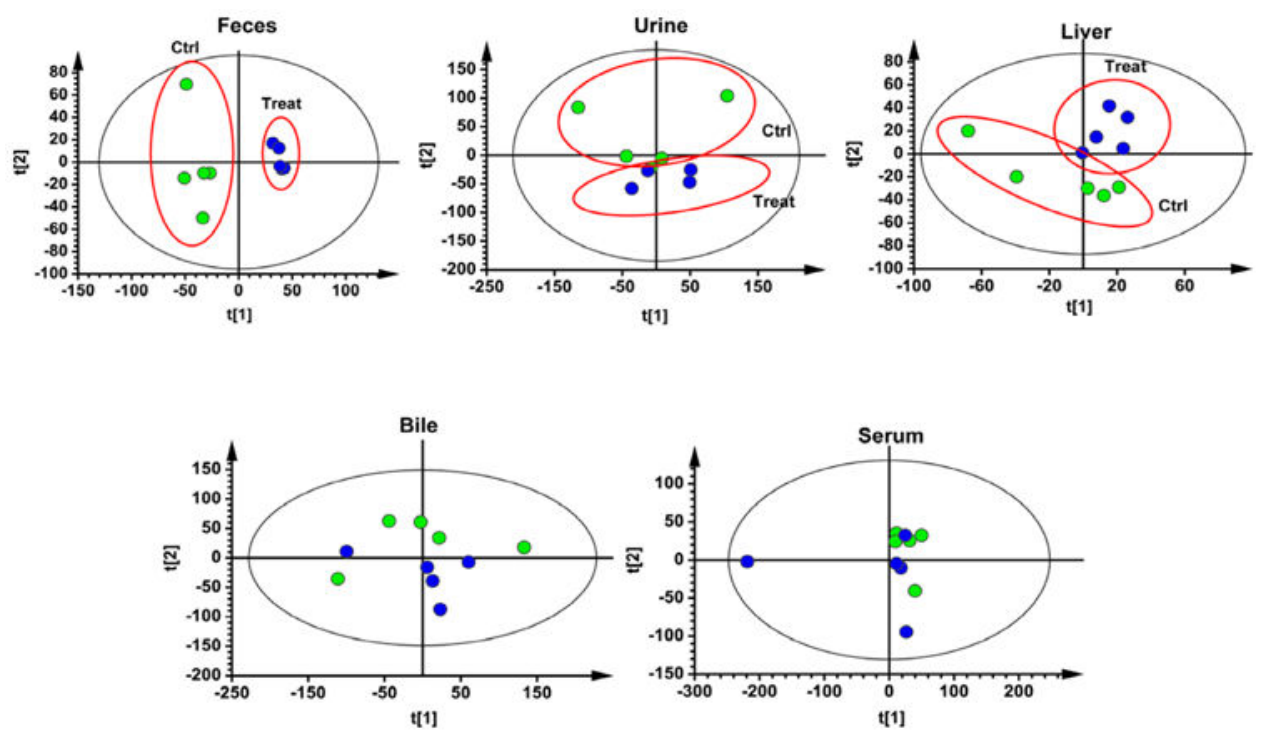

Fig. 2.

Metabolomics analysis of feces, urine, liver, bile and serum samples obtained from control and MRS5980-treated groups. Unsupervised principal components analysis (PCA) was utilized, and PCA scoring plots were given. The green and blue dots represent control and MRS5980-treated groups, respectively. $\mathrm{N}=5$ in each group. 


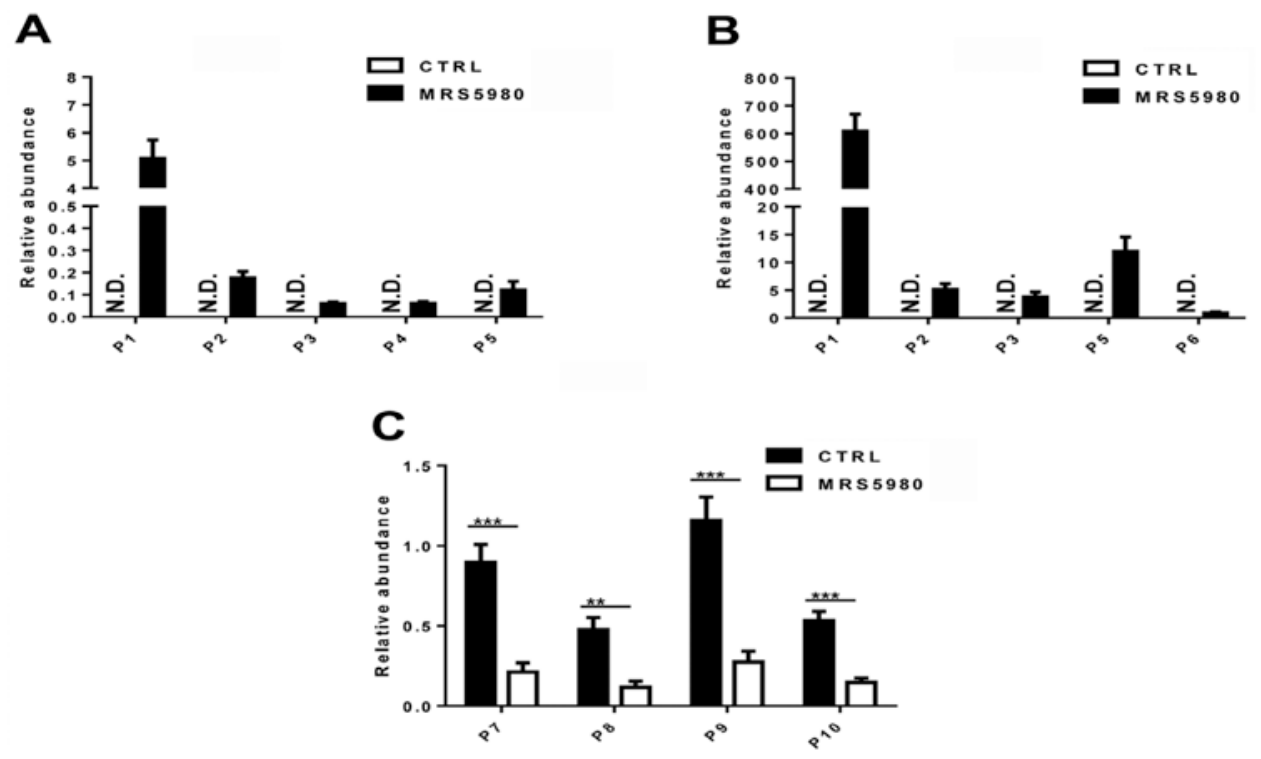

Fig. 3.

Comparison of ion abundances in urine (A), feces (B) and liver (C) between control and MRS5980-treated mice. Data were given as mean plus S.D ( $\mathrm{n}=5)$. N.D., not detected; **, $\mathrm{p}<0.01 ; * * *, \mathrm{p}<0.001$. 

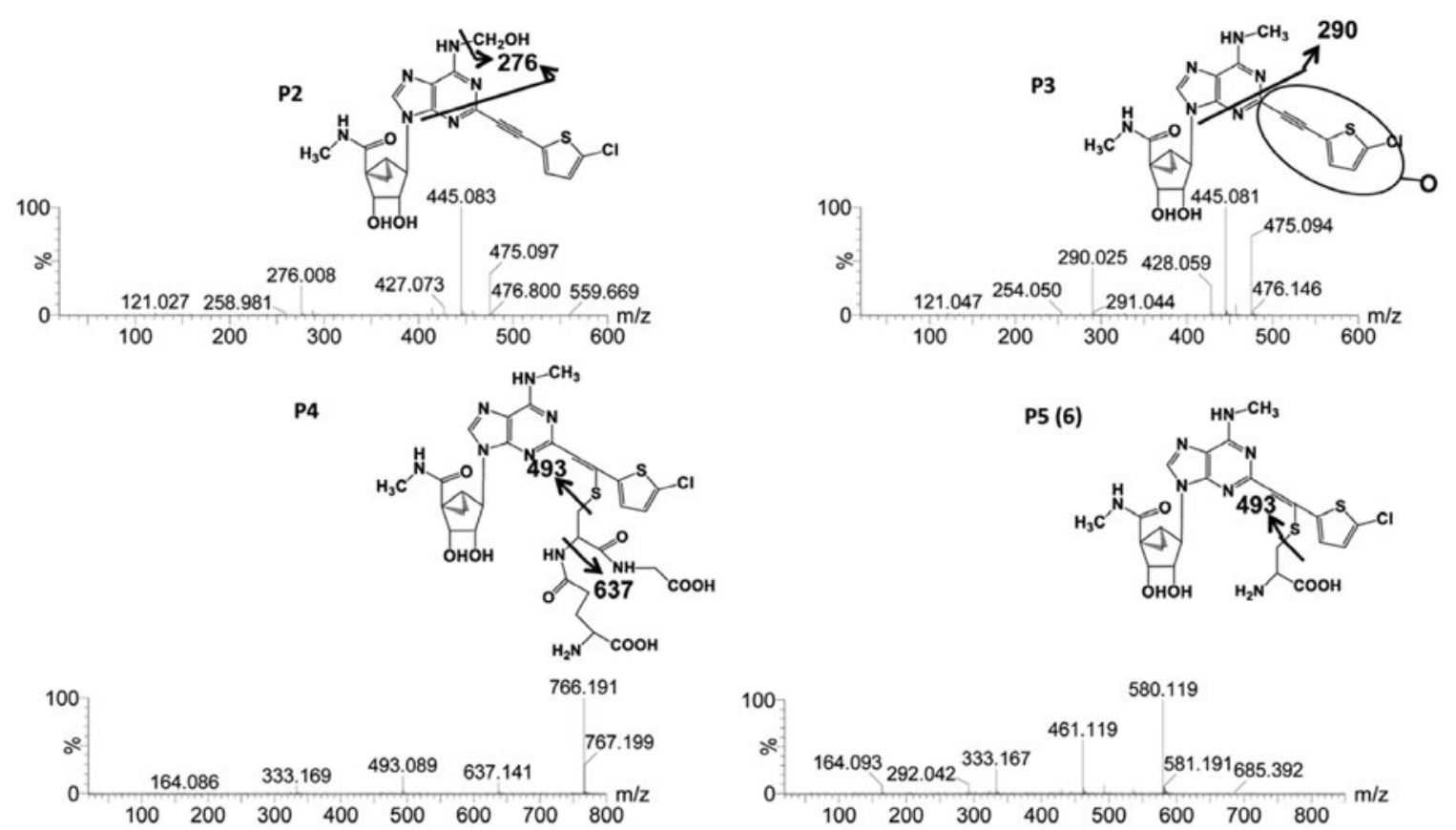

Fig. 4.

Tandem MS spectra and proposed chemical structures of P2, P3, P4 and P5 (6). P2, P3, P4 and P5 (6) represent oxidized MRS5980, oxidized MRS5980, MRS5980 GSH conjugate and MRS5980 cysteine conjugate, respectively. 


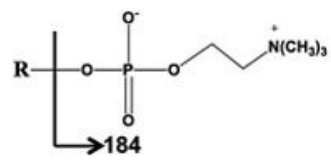

P7

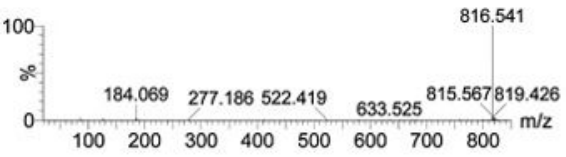

P9

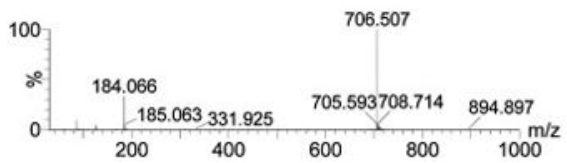

P8

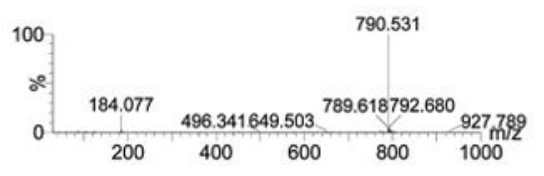

P10

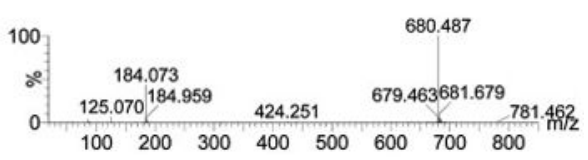

Fig. 5.

Tandem MS spectra and proposed chemical structures of metabolites P7-P10. These four ions were proposed to be PC components according to the metabolomics database and typical fragment ion 184. 
A

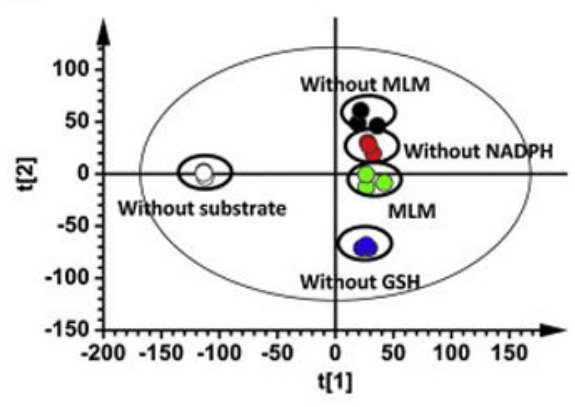

C

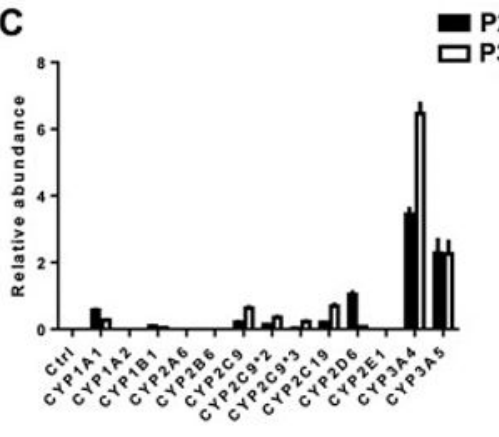

B

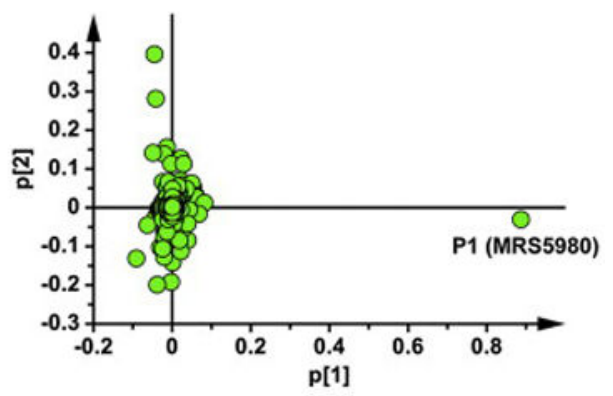

Fig. 6.

Determination of in vitro metabolism and electrophilic reactivity of MRS5980. The in vitro incubation system contained $50 \mathrm{mM}$ Tris- $\mathrm{HCl}$ buffer ( $\mathrm{pH}=7.4), 0.5 \mathrm{mg} \mathrm{mL}^{-1} \mathrm{MLM}, 5 \mathrm{mM}$ $\mathrm{MgCl}_{2}, 100 \mu \mathrm{M}$ MRS5980, $2.5 \mathrm{mM}$ GSH and $1 \mathrm{mM}$ NADPH. The incubation mixtures without GSH, MLM (or HLM), NADPH and MRS5980 were used as the control. (A) Unsupervised principal components analysis (PCA) of MLM incubation system and control system. (B) PCA loading plot; (C) Screening of the recombinant CYP isoforms involved in the metabolic elimination of MRS5980. 

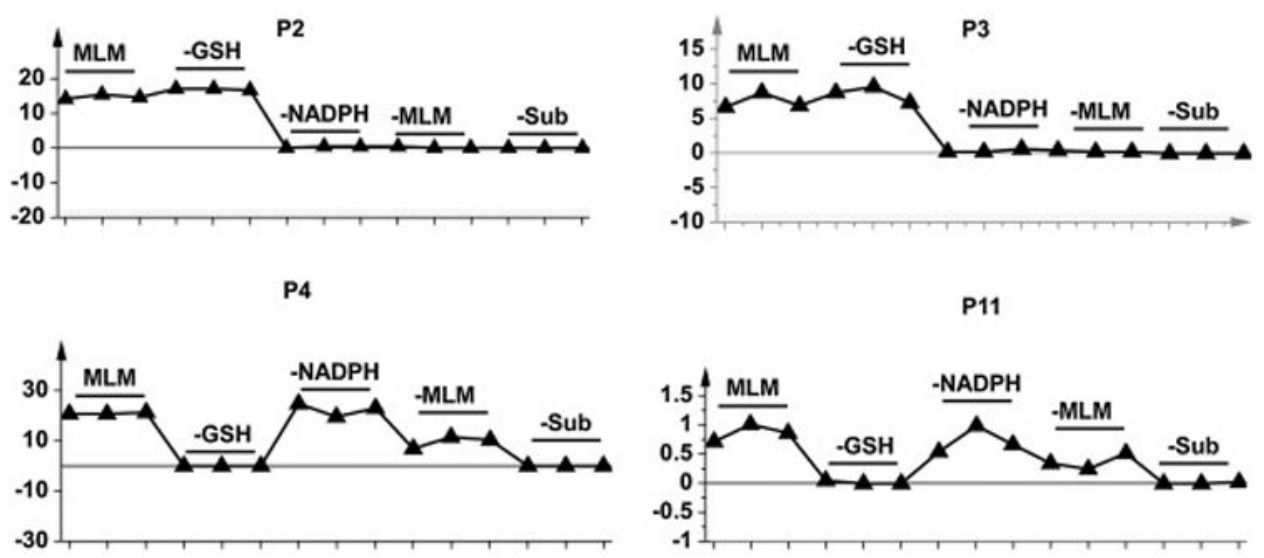

Fig. 7.

Trend plot for the formation of oxidized metabolites of MRS5980 (P2 and P3) and GSH conjugates (P4 and P11) in the mouse liver microsome (MLM) incubation system and the incubation system without MLM (-MLM), substrate (-Sub), NADPH (-NADPH), and GSH (-GSH). 
P2

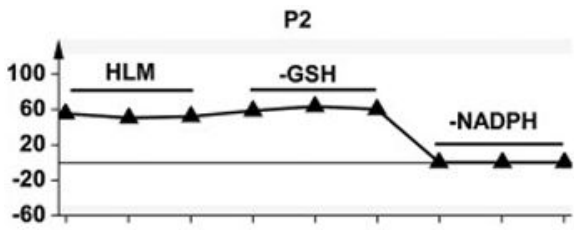

P4

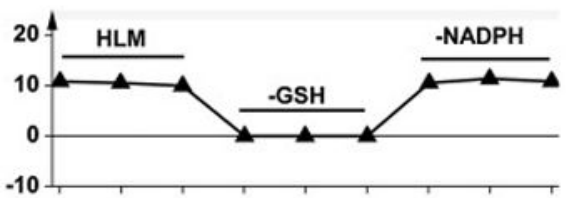

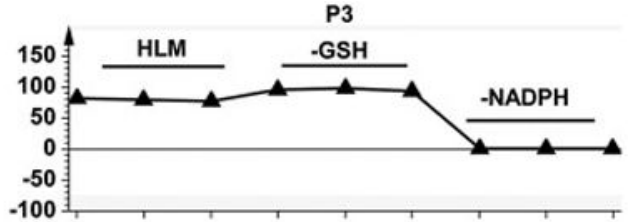

P11

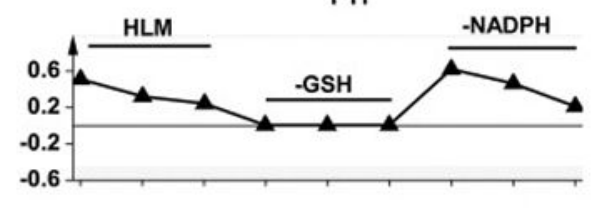

P12

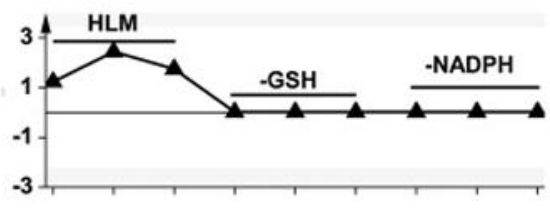

Fig. 8.

Trend plot for the formation of oxidized metabolites of MRS5980 (P2 and P3), GSH conjugates (P4 and P11), and a new GSH conjugate (P12) in the human liver microsome (MLM) incubation system and the incubation system without HLM (-HLM), NADPH (NADPH), and GSH (-GSH). 


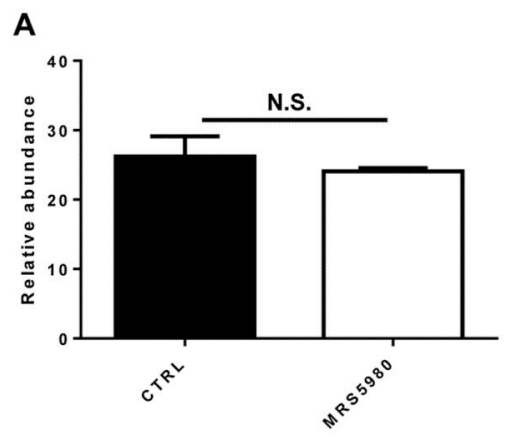

B

A

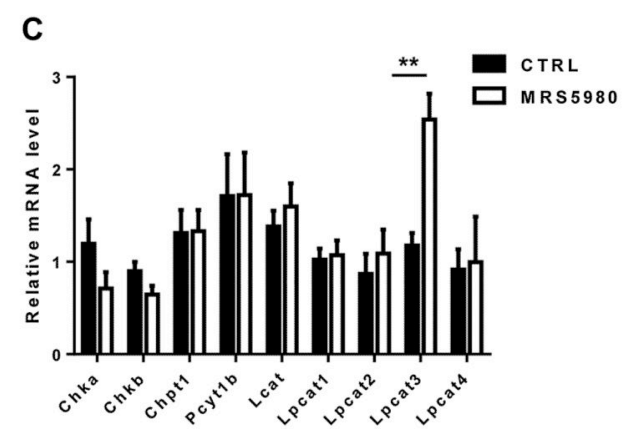

CTRL
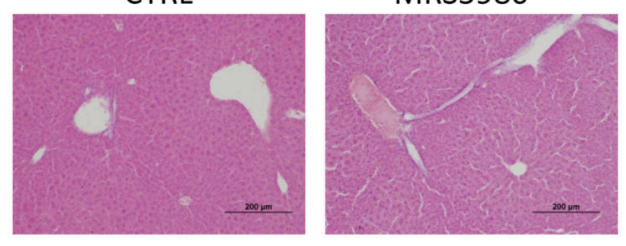

Fig. 9.

In vivo effects of MRS5980 on liver histology, GSH and lipid genes expression. (A) GSH level in the liver from the control and MRS5980-treated group. N.S., not significant. (B) Histological analysis of liver samples from control and MRS5980-treated groups. Control mice were treated with corn oil only. (C) Comparison of gene expression related to lipid metabolism. **, $\mathrm{p}<0.01$. 

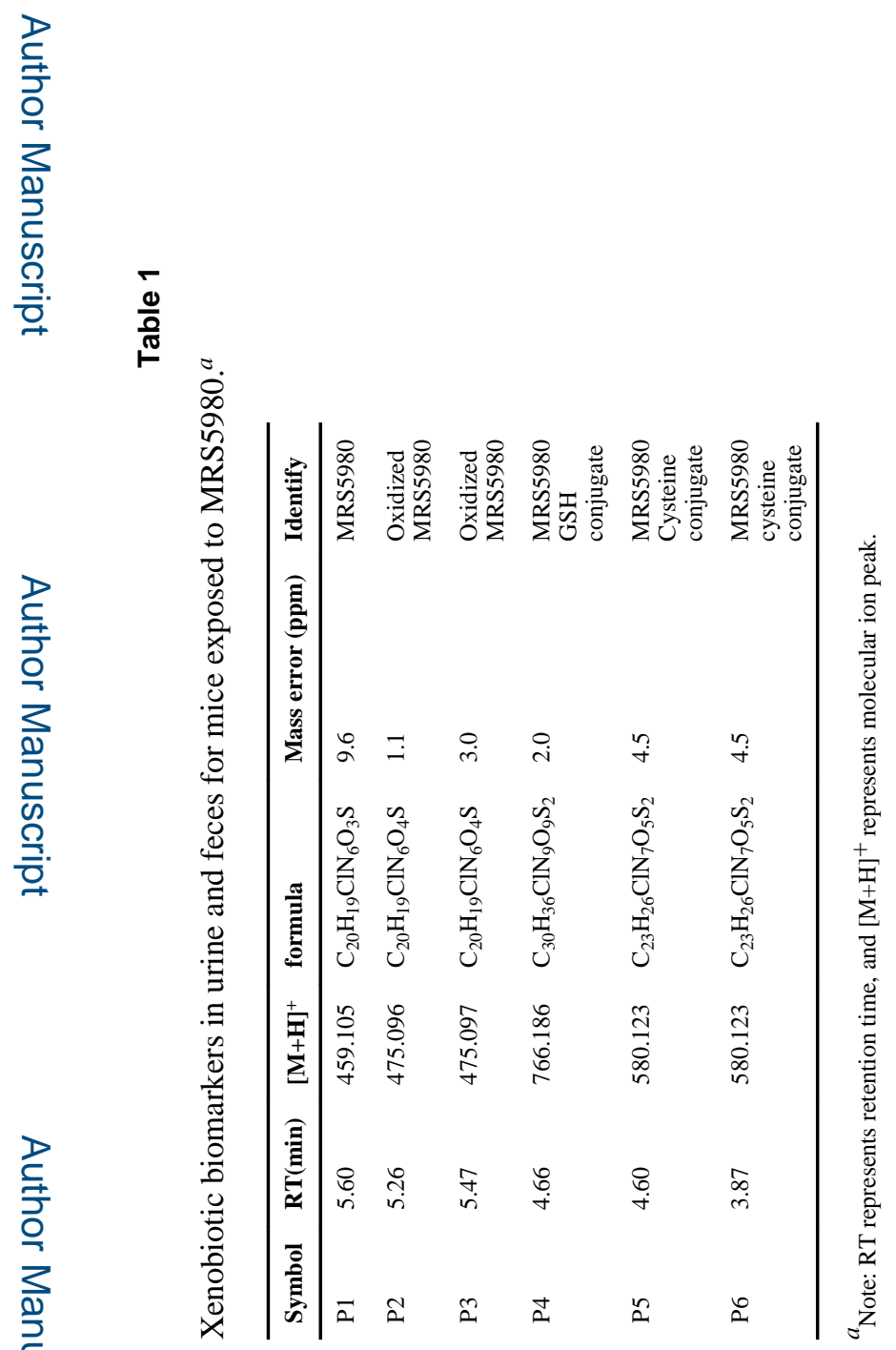

Biochem Pharmacol. Author manuscript; available in PMC 2016 September 15. 